\title{
Eliza Wajch
}

Small systems - on approximation of compact sets of measurable functions to compact subsets of $C_{c-o}(X)$

Czechoslovak Mathematical Journal, Vol. 41 (1991), No. 4, 619-633

Persistent URL: http://dml.cz/dmlcz/102494

\section{Terms of use:}

(C) Institute of Mathematics AS CR, 1991

Institute of Mathematics of the Czech Academy of Sciences provides access to digitized documents strictly for personal use. Each copy of any part of this document must contain these Terms of use.

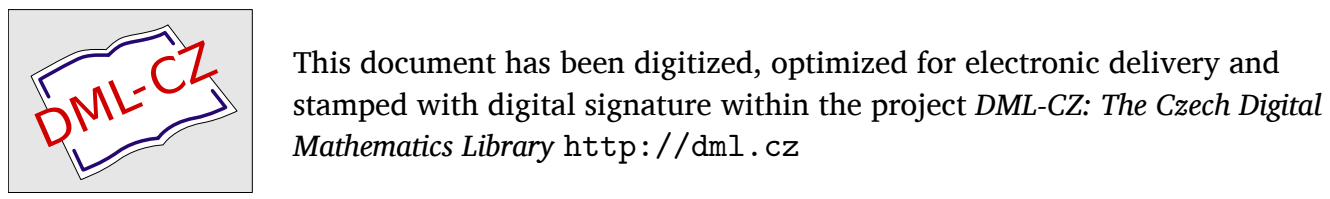




\section{SMALL SYSTEMS - ON APPROXIMATION OF COMPACT SETS OF MEASURABLE FUNCTIONS TO COMPACT SUBSETS$$
\text { OF } C_{\mathrm{c}-\mathrm{o}}(X)
$$

Eliza WaJch, Lódż

(Received July 12, 1989)

It was realized in $[3,10,13,17,18,21,25]$ that a great number of theorems in measure theory can be formulated in terms of some systems of "small" sets, without using any measure. After extending the notion of the convergence with respect to a measure to the convergence with respect to a small system (cf. [13] and [18]), the classical theorem of Fréchet on compact sets of measurable functions (cf. $[2,6,9]$ ) was generalized in [10] to sets of measurable functions, compact in the sense of the convergence with respect to a small system. Some connections between sets of Borel functions which are compact in the sense of the convergence with respect to an upper semicontinuous small system of Borel subsets of a perfectly normal compact space and uniformly compact sets of continuous functions were brought out in [25]. The present paper, being a continuation of [25], deals with an upper semicontinuous small system $\left(\sigma_{n}\right)$ on a $\sigma$-algebra $\mathscr{M}$ containing the $\sigma$-algebra of Baire sets of a Tychonoff $k$-space $X$. The main purpose of this article is to answer the question: Under what assumptions on $\mathscr{M},\left(\sigma_{n}\right)$ or $X$ is an arbitrary family $\Phi$ of $\mathscr{M}$-measurable almost everywhere finite real functions on $X$ compact in the sense of the convergence with respect to $\left(J_{n}\right)$ if and only if the following property:

"For any positive integer $n$, there exists a set $\Phi^{*}$ of continuous real functions on $X$, being compact in the compact-open topology and such that, whenever $f \in \Phi$, one can find an $f^{*} \in \Phi^{*}$ with $\left\{x \in X: f(x) \neq f^{*}(x)\right\} \in \delta_{n} "$ holds?

Similarly as [25], the theorems obtained here generalize Kisyński's result of [15; Th. 1, p. 228]. Moreover, they lead to many problems which seem to be open and will be listed at the end of the article. Let us mention that if $\mu$ is a finite $\sigma$-additive measure on $\mathscr{M}$, and $\jmath_{n}=\{A \in \mathscr{M}: \mu(A) \leqq 1 / n\}$ for $n \in N$, then $\left(\sigma_{n}\right)$ forms an upper semicontinuous small system on $\mathscr{M}$; thus, various conclusions for finite measures can easily be drawn from this work.

0. Preliminaries. Let $\mathscr{M}$ be a $\sigma$-algebra of subsets of a nonempty set $X$. Suppose we are given a sequence $\left(s_{n}\right)$ of nonempty subfamilies of $\mathscr{M}$ which satisfies the following conditions: 
(I) for any $n \in N$, there exists a sequence $\left(k_{i}\right)$ of positive integers such that if $A_{i} \in s_{k_{i}}$ for $i \in N$, then $\bigcup_{i=1}^{\infty} A_{i} \in s_{n}$;

(II) for any $n \in N, A \in \varsigma_{n}$ and $B \in \mathscr{M}$ such that $B \subset A$, we have $B \in \circlearrowleft_{n}$;

(III) for any $n \in N, A \in \delta_{n}$ and $B \in \bigcap_{i=1}^{\infty} J_{i}$, we have $A \cup B \in \sigma_{n}$;

(IV) $j_{n} \supset \jmath_{n+1}$ for each $n \in N$.

The sequence $\left(j_{n}\right)$ is said to be a small system on $\mathscr{M}($ cf. $[3,10,13,17,18,21,25])$. If, in addition, $\left({ }_{n}\right)$ has the property

(V) for any $n \in N$ and any nonincreasing sequence $\left(A_{i}\right)$ of members of $\mathscr{M} \backslash \circlearrowleft_{n}$, we have $\bigcap_{i=1}^{\infty} A_{i} \neq \emptyset$,

then it is called an upper semicontinuous (abbr. u.s.c.) small system (cf. [17; Def. 18.29]).

Let us put $\lrcorner=\bigcap_{n=1}^{\infty}{ }_{n}$. Obviously, $\lrcorner$ forms a $\sigma$-ideal on $\mathscr{M}$. One says that a property holds $j$-almost everywhere (abbr. s-a.e.) on $X$ if the set of points not having this property belongs to $J$. Denote by $F[\mathscr{M}, \jmath]$ the family of all $s$-a.e. finite $\mathscr{M}$-measurable real functions defined on $X$.

0.1. Definition (cf. [13] and [18]). A sequence $\left(f_{n}\right)$ of functions from $F[\mathscr{M}, 0]$ converges with respect to the small system $\left(\sigma_{n}\right)$ to a function $f \in F[\mathscr{M}, \jmath]$ if, for any $\varepsilon>0$ and any $m \in N$, there exists $n_{0} \in N$ such that

$$
\left\{x \in X:\left|f_{n}(x)-f(x)\right|>\varepsilon\right\} \in \jmath_{m}
$$

whenever $n \geqq n_{0}$.

0.2. Definition (cf. [10]). A family $\Phi \subset F[\mathcal{M}, \mathcal{\jmath}]$ is called:

(a) compact in the sense of the convergence with respect to the small system $\left(\sigma_{n}\right)$ (abbr. $\left(\sigma_{n}\right)$-compact) if each sequence of functions from $\Phi$ contains a subsequence converging with respect to $\left(j_{n}\right)$ to some function from $F[\mathscr{M}, 0]$;

(b) compact in the sense of the convergence with respect to the $\sigma$-ideal s (abbr. $s$-compact) if each sequence of functions from $\Phi$ contains a subsequence converging $s$-a.e. on $X$ to some function from $F[\mathscr{M}, \mathrm{J}]$.

It was observed in $[10]$ that $s$-compactness is equivalent to $\left(\sigma_{n}\right)$-compactness if and only if $\left(\sigma_{n}\right)$ is u.s.c. Further, we shall apply the following characterization of $\left(\sigma_{n}\right)$ compactness obtained in [10]:

0.3. Theorem. Suppose $\left(\diamond_{n}\right)$ is u.s.c. A family $\Phi \subset F\left[\mathscr{M}, \sigma^{\circ}\right.$ is $\left(\diamond_{n}\right)$-compact if and only if it satisfies the following two conditions:

(a) for any $n \in N$, there exists a positive integer $t$ such that $\{x \in X:|f(x)|>t\} \in \delta_{n}$ whener $f \in \Phi\left(\right.$ i.e. $\Phi$ is $\left(\diamond_{n}\right)$-equibounded);

(b) for any $\varepsilon>0$ and any $n \in N$, there exist a finite subfamily $\mathscr{P}$ of $\mathscr{M}$ and a collection $\left\{A_{f}: f \in \Phi\right\} \subset \varsigma_{n}$, such that $\bigcup\{P: P \in \mathscr{P}\}=X$ and, for any $P \in \mathscr{P}$ and 
$f \in \Phi$, we have $|f(x)-f(y)| \leqq \varepsilon$ whenever $x, y \in P \backslash A_{f}$ (i.e. $\Phi$ is $\left(j_{n}\right)$-equimeasurable).

From now on, $X$ will be a Tychonoff $k$-space, unless specified otherwise. The symbol $C_{\mathrm{c}-\mathrm{o}}(X)$ will stand for the algebra of all continuous finite real functions defined on $X$, with the compact-open topology. By $\mathscr{G}(X), \mathscr{F}(X), \mathscr{G}^{*}(X)$ and $\mathscr{F}^{*}(X)$ we shall denote the families of all open, closed, cozero and zero subsets of $X$, respectively. The Borel $\sigma$-algebra $\mathscr{B}(X)$ in $X$ is the smallest $\sigma$-algebra containing $\mathscr{G}(X)$, and the Baire $\sigma$-algebra $\mathscr{B}^{*}(X)$ in $X$ is the $\sigma$-algebra generated by $\mathscr{G}^{*}(X)$. Of course, $\mathscr{B}^{*}(X) \subset \mathscr{B}(X)$; however, the equality $\mathscr{B}^{*}(X)=\mathscr{B}(X)$ need not hold. Other symbols not defined here are standard. All topological notions used in this paper can be found in $[1,5,8,22]$.

In the sequel, we shall always assume on the $\sigma$-algebra $\mathscr{M}$ that it contains $\mathscr{B}^{*}(X)$; moreover, $\left(j_{n}\right)$ will be a fixed u.s.c. small system on $\mathscr{M}$. Then $C_{\mathrm{c}-\mathrm{o}}(X) \subset F[\mathscr{M}, j]$ (recall that $j=\bigcap_{n=1}^{\infty} j_{n}$ ).

For a family $\Phi \subset F[\mathscr{U}, \jmath]$, denote by $T(\Phi)$ and $T_{\lrcorner}(\Phi)$ the following statements: $T(\Phi)$ : If $\Phi$ is $\left(j_{n}\right)$-compact, then, for any $n \in N$, there exists a compact set $\Phi^{*} \subset$ $\subset C_{\mathrm{c}-\mathrm{o}}(X)$ such that, whenever $f \in \Phi$, there is an $f^{*} \in \Phi^{*}$ with $\{x \in X$ : $\left.f(x) \neq f^{*}(x)\right\} \in v_{n}$.

$T_{\jmath}(\Phi)$ : If $\Phi$ is $j$-compact, then there exists a sequence $\left(\Phi_{n}^{*}\right)$ of compact subsets of $C_{\mathrm{c}-\mathrm{o}}(X)$ with the property that, for any sequence $\left(f_{n}\right)$ of functions from $\Phi$, there exists a sequence $\left(f_{n}^{*}\right)$ of continuous functions such that $\bigcap^{\infty} \bigcup^{\infty}\{x \in X$ : $\left.f_{n}(x) \neq f_{n}^{*}(x)\right\} \in \jmath$ and $f_{n}^{*} \in \Phi_{n}^{*}$ for any $n \in N$.

Let $T^{-1}(\Phi)$ and $T_{\mathcal{J}}^{-1}(\Phi)$ denote the implications converse to $T(\Phi)$ and $T_{\lrcorner}(\Phi)$, respectively.

As it has already been mentioned, $j$-compactness is equivalent to $\left(i_{n}\right)$-compactness because $\left(J_{n}\right)$ is assumed to be u.s.c., so one can expect that the following theorem holds:

0.4. Theorem. $T(\Phi) \Leftrightarrow T_{\jmath}(\Phi)$ and $T^{-1}(\Phi) \Leftrightarrow T_{\diamond}^{-1}(\Phi)$ for any $\Phi \subset F[\mathscr{M}, 0]$.

Proof. Consider any $\Phi \subset F[\mathscr{M}, 0]$. By Lemma 1. of [18], there exists a sequence $\left(k_{n}\right)$ of positive integers such that if $A_{n} \in \delta_{k_{n}}$ for $n \in N$, then $\bigcup_{n=m}^{\infty} A_{n} \in \delta_{m}$ for any $m \in N$. Suppose that, for any $n \in N$, there exists a compact set $\Phi_{n}^{*} \subset C_{\mathrm{c}-\mathrm{o}}(X)$ with the property that, whenever $f \in \Phi$, we can find an $f^{*} \in \Phi_{n}^{*}$ such that $\{x \in X: f(x) \neq$ $\left.\neq f^{*}(x)\right\} \in j_{k_{n}}$. Let $\left(f_{n}\right)$ be any sequence of functions from $\Phi$. For each $n \in N$, choose $f_{n}^{*} \in \Phi_{n}^{*}$ such that $\left\{x \in X: f_{n}(x) \neq f_{n}^{*}(x)\right\} \in j_{k_{n}}$. Then the set $\bigcap_{m=1}^{\infty} \bigcup_{n=m}^{\infty}\left\{x \in X: f_{n}(x) \neq\right.$ $\left.\neq f_{n}^{*}(x)\right\}$ belongs to $s$.

Conversely, assume that $\left(\Phi_{n}^{*}\right)$ is a sequence of compact subsets of $C_{\mathrm{c}-\mathrm{o}}(X)$ satisfying the condition given in $T_{s}(\Phi)$, and suppose that there exists $n_{0} \in N$ having the property that, for any $n \in N$, there is an $f_{n} \in \Phi$ such that $\left\{x \in X: f_{n}(x) \neq f^{*}(x)\right\} \notin \sigma_{n_{0}}$ whenever 
$f^{*} \in \Phi_{n}^{*}$. Let $f_{n}^{*} \in \Phi_{n}^{*}$ be such that the set $A=\bigcap_{m=1}^{\infty} \bigcup_{n=m}^{\infty}\left\{x \in X: f_{n}(x) \neq f_{n}^{*}(x)\right\}$ belongs to $s$. Since $\bigcup_{n=m}^{\infty}\left\{x \in X: f_{n}(x) \neq f_{n}^{*}(x)\right\} \notin \sigma_{n_{0}}$ for any $m \in N$, the u.s.c. of $\left(\jmath_{n}\right)$ implies that $A \notin J$. The contradiction obtained, together with the equivalence of $\left(J_{n}\right)$-compactness and $\jmath$-compactness, completes the proof.

It follows from the above theorem that if $\mathscr{L}$ is a $\sigma$-ideal on $\mathscr{M}$ such that $T_{\mathscr{L}}\left(\Phi_{0}\right)$ $\left(T_{\mathscr{L}}^{-1}\left(\Phi_{0}\right)\right.$, resp.) holds for some $\Phi_{0} \subset F[\mathscr{M}, \mathscr{L}]$, then $T\left(\Phi_{0}\right)\left(T^{-1}\left(\Phi_{0}\right)\right.$, resp.) holds for each u.s.c. small system $\left(\varsigma_{n}\right)$ on $\mathscr{M}$ such that $\mathscr{L}=\bigcap_{n=1}^{\infty} \varsigma_{n}$. As was observed in [18], there exist $\sigma$-ideals which are not the intersection of any u.s.c. small system.

0.5. Remark. Let us put $\jmath^{\wedge}=\{A \subset X$ : there exists $B(A) \in\lrcorner$ with $\left.A \subset B(A)\right\}$, $\hat{\sigma}_{n}^{\wedge}=\left\{(A \backslash B) \cup C: A \in \sigma_{n}\right.$ and $\left.B, C \in \sigma^{\wedge}\right\}$ for $n \in N$, and $\mathscr{M}^{\wedge}=\{(A \backslash B) \cup C$ : $A \in \mathscr{U}$ and $\left.B, C \in,^{\wedge}\right\}$ (in other words, $\mathscr{M}^{\wedge}$ is the smallest $\sigma$-algebra which contains both $\mathscr{U}$ and $\left.\delta^{\wedge}\right)$. We shall call $\mathscr{M}^{\wedge},\left(\sigma_{n}^{\wedge}\right)$ and $\sigma^{\wedge}$ the completions of $\mathscr{M},\left(\sigma_{n}\right)$ and $s$, respectively. Without any difficulties one can check that (\lrcorner$\left._{n}^{\wedge}\right)$ is an u.s.c. small system on $\mathscr{U}^{\wedge}$ such that $\left.\jmath^{\wedge}=\bigcap_{n=1}^{\infty}\right\lrcorner_{n}^{\wedge}$; furthermore, for any $\left.f \in F\left[\mathscr{M}^{\wedge},\right\lrcorner^{\wedge}\right]$, there exists $g \in F[\mathscr{M}, j]$ with $\{x \in X: f(x) \neq g(x)\} \in \jmath^{\wedge}$. Therefore, $T_{\lrcorner}(\Phi)\left({T_{\lrcorner}}^{-1}(\Phi)\right.$, resp. $)$ is true for any $\Phi \subset F\left[\mathscr{M}^{\wedge}, s^{\wedge}\right]$ if and only if $T_{s}(\Phi)\left(T_{\triangleleft}^{-1}(\Phi)\right.$, resp.) is true for any $\Phi \subset F[\mathscr{l l}, j]$.

In section 1 we shall give a necessary condition and a number of sufficient conditions for $\mathscr{U},\left(j_{n}\right)$ and $X$ in order that $T(\Phi)$ be true for any $\Phi \subset F[\mathscr{M}, \jmath]$, whereas section 2 will be devoted to sufficient conditions for $\mathscr{M},\left(\diamond_{n}\right)$ and $X$ in order that $T^{-1}(\Phi)$ hold for any $\Phi \subset F[\mathscr{M}, \jmath]$.

1. The implication $T(\Phi)$. The symmetric difference of sets $A$ and $B$ will be denoted by $A \Delta B$.

1.1. Defiaition. The small system $\left(\sigma_{n}\right)$ is called:

(a) regular ((*)-regular, respectively) if, for any $A \in \mathscr{M}$ and $n \in N$, there exists $F \in \mathscr{F}(X) \cap \mathscr{U}\left(F \in \mathscr{F}^{*}(X)\right.$, respectively) such that $F \subset A$ and $A \backslash F \in \sigma_{n}$;

(b) weakly regular (weakly (*)-regular, respectively) if, for any $A \in \mathscr{M}$ and $n \in N$, there exists $F \in \mathscr{F}(X) \cap \mathscr{M}\left(F \in \mathscr{F}^{*}(X) \text {, respectively) such that } A \Delta F \in\right\lrcorner_{n}$.

1.2. Example. Let us observe that if $\mu$ is a two-valued Borel probability on $X$, then putting $y_{n}=\{A \in \mathscr{B}(X): \mu(A)=0\}$ for any $n \in N$, we obtain a weakly (*)-regular u.s.c. small system $\left(\varsigma_{n}\right)$ on $\mathscr{B}(X)$ (for any $A \in \mathscr{B}(X)$, it suffices to consider $F^{-}=X$ if $\mu(A)=1$ and $F=\emptyset$ otherwise); however, $\left(\sigma_{n}\right)$ need not be regular. Indeed, the Dieudonné measure on $\omega_{1}+1$ equipped with the order topology is a two-valued Borel probability on $\omega_{1}+1$ which fails to be regular (cf. [7; Example 5.5]).

1.3. Proposition. (a) The small system $\left(\sigma_{n}\right)$ is regular $((*)$-regular, respectively) if and only if, for any $A \in \mathscr{M}$ and $n \in N$, there exist $F \in \mathscr{F}(X) \cap \mathscr{M}$ and $G \in \mathscr{G}(X) \cap$ 
$\cap \mathscr{U}\left(F \in \mathscr{F}^{*}(X)\right.$ and $G \in \mathscr{G}^{*}(X)$, respectively) such that $F \subset A \subset G$ and $G \backslash F \in \delta_{n}$.

(b) The small system $\left(j_{n}\right)$ is weakly regular (weakly $(*)$-regular, respectively) if and only if, for any $A \in \mathscr{M}$ and $n \in N$, there exists $G \in \mathscr{G}(X) \cap \mathscr{M}\left(G \in \mathscr{G}^{*}(X)\right.$, respectively) such that $A \Delta G \in J_{n}$.

Proof. Let us fix $A \in \mathscr{I}$ and $n_{0} \in N$.

(a) Suppose that $\left(s_{n}\right)$ is regular $((*)$-regular, respectively). There exists $m \in N$ such that $B \cup C \in \nu_{n_{0}}$ whenever $\left.B, C \in\right\lrcorner_{m}$. Choose sets $F, H \in \mathscr{F}(X) \cap \mathscr{M}(F, H \in$ $\in \mathscr{F}^{*}(X)$, respectively) such that $F \subset A, H \subset X \backslash A, A \backslash F \in \sigma_{m}$ and $(X \backslash A) \backslash H \in \sigma_{m}$. If $G=X \backslash H$, then $A \subset G$ and $G \backslash F \subset[(X \backslash A) \backslash H] \cup(A \backslash F)$, so $G \backslash F \in{ }_{n_{0}}$.

(b) Suppose $\left(o_{n}\right)$ is weakly regular (weakly $(*)$-regular, resp.). Let us take a set $F \in \mathscr{F}(X) \cap \mathscr{U}\left(F \in \mathscr{F} *(X)\right.$, respectively) such that $(X \backslash A) \Delta F \in \sigma_{n_{0}}$. Then, for $G=X \backslash F$, we have $A \Delta G \in j_{n_{0}}$.

1.4. Lemma. If $\mathscr{A}$ is the collection of all sets $A \in \mathscr{M}$ having the property that, for any $n \in N$, there exist $F \in \mathscr{F}^{*}(X)$ and $G \in \mathscr{G}^{*}(X)$ such that $F \subset A \subset G$ and $G \backslash F \in j_{n}$, then $\mathscr{A}$ forms a $\sigma$-algebra containing $\mathscr{B}^{*}(X)$.

Proof. Clearly, $\mathscr{A}$ is stable under complements. To show that it is stable under countable unions, let us fix $n_{0} \in N$ and take a sequence $\left(k_{i}\right)$ of positive integers such that $\bigcup_{i=1}^{\infty} E_{i} \in j_{n_{0}}$ whenever $E_{i} \in J_{k_{i}}$ for $i \in N$. Assume that $A_{i} \in \mathscr{A}$ for $i \in N$. There exist sets $F_{i} \in \mathscr{F}^{*}(X)$ and $G_{i} \in \mathscr{G}^{*}(X)$ such that $F_{i} \subset A_{i} \subset G_{i}$ and $G_{i} \backslash F_{i} \in \sigma_{k_{i+1}}$ for $i \in N$. It follows from the u.s.c. of $\left(o_{n}\right)$ that there is an $m_{0} \in N$ for which $\left(\bigcup_{i=1}^{\infty} F_{i}\right) \backslash$ $\backslash\left(\bigcup_{i=1}^{m_{0}} F_{i}\right) \in J_{k_{1}}$. Put $G=\bigcup_{i=1}^{\infty} G_{i}$ and $F=\bigcup_{i=1}^{m_{0}} F_{i}$. Obviously, $G \in \mathscr{G}^{*}(X) \underset{\infty}{\text { and }} F \in \mathscr{F} *(X)$ (cf. $\left[8 ; 1.10\right.$ and 1.14]); moreover, $F \subset \bigcup_{i=1}^{\infty} A_{i} \subset G$ and $G \backslash F \subset \bigcup_{i=1}^{\infty}\left(G_{i} \backslash F_{i}\right) \cup$ $\cup \bigcup_{i=1}\left(F_{i} \backslash F\right)$, so $G \backslash F \in j_{n_{0}}$.

Now, let us observe that if $f \in C_{\mathrm{c}-\mathrm{o}}(X)$, then $f^{-1}(R \backslash\{0\})=\bigcup_{i=1}^{\infty} f_{i}^{-1}(0)$ where $f_{i}=\min \{|f|-(1 / i), 0\}$ for $i \in N$; hence the u.s.c. of $\left(\sigma_{n}\right)$ implies that $f^{-1}(R \backslash\{0\}) \backslash$ $\backslash \bigcup_{i=1}^{m} f_{i}^{-1}(0)$ belongs to $j_{n_{0}}$ for some $m \in N$. Consequently, $\mathscr{B}^{*}(X) \subset \mathscr{A}$.

1.5. Remark. With Lemma 1.4 in hand, it is not difficult to show that $\left(\delta_{n}\right)$ is weakly (*)-regular ((*)-regular, respectively) if and only if, for any $A \in \mathscr{M}$ and $n \in N$, there exists $B \in B^{*}(X)$ such that $A \Delta B \in o_{n}$ (resp. $B \subset A$ and $A \backslash B \in \delta_{n}$ ).

1.6. Lemma. Suppose $\left(s_{n}\right)$ is weakly $(*)$-regular. If $\Phi \subset F[\mathscr{M}, 0]$ is $\left(s_{n}\right)$-compact, then, for any $\varepsilon>0$ and $n_{0} \in N$, there exist a collection $\left\{A_{f}: f \in \Phi\right\} \subset \sigma_{n_{0}}$, a continuous function $h: X \rightarrow[0,1]$ and a real number $\delta>0$, such that, for any $f \in \Phi$ and $x, y \in X \backslash A_{f}$, we have $|f(x)-f(y)| \leqq \varepsilon$ whenever $|h(x)-h(y)| \leqq \delta$.

Proof. Let us fix $\varepsilon>0$ and $n_{0} \in N$. Choose a sequence $\left(k_{i}\right)$ of positive integers 
such that if $A_{i} \in J_{k_{i}}$ for $i \in N$, then $\bigcup_{i=1}^{\infty} A_{i} \in J_{n_{0}}$. By Theorem 0.3, there exist collections $\left\{P_{1}, \ldots, P_{m}\right\} \subset \mathscr{M}$ and $\left\{E_{f}: f \in \Phi\right\} \subset J_{k_{1}}$, such that $\bigcup_{i=1}^{m} P_{i}=X$ and, for any $f \in \Phi$ and $i=1, \ldots, m$, we have $|f(x)-f(y)| \leqq \varepsilon$ whenever $x, y \in P_{i} \backslash E_{f}$. It follows from the weak $(*)$-regularity of $\left(j_{n}\right)$ that there exist sets $F_{i} \in \mathscr{F}^{*}(X)$ such that $P_{i} \Delta F_{i} \in$ $\in j_{k_{i+1}}$ for $i=1, \ldots, m$. Put $B_{1}=F_{1}$ and $B_{i}=F_{i} \backslash \bigcup_{k=1} F_{k}$ for $i=1, \ldots, m$. According to Lemma 1.4, there exist $Z_{i} \in \mathscr{F}^{*}(X)$ such that $Z_{i} \subset B_{i}$ and $B_{i} \backslash Z_{i} \in \sigma_{k_{i+m+1}}$ for $i=1, \ldots, m$. Let $A_{f}=E_{f} \cup \bigcup_{i=1}^{m}\left(P_{i} \Delta F_{i}\right) \cup \bigcup_{i=1}^{m}\left(B_{i} \backslash Z_{i}\right)$ for $f \in \Phi$. Clearly, $\left\{A_{f}: f \in \Phi\right\} \subset j_{n_{0}}$. Since any two disjoint members of $\mathscr{F} *(X)$ are completely separated and $\mathscr{F}^{*}(X)$ is closed under finite unions (cf. $[8 ; 1.10$ and 1.15$\left.]\right)$, there exists a continuous function $h: X \rightarrow[0,1]$ such that $h\left(Z_{i}\right) \subset\{1 / i\}$ for $i=1, \ldots, m$. Let us put $\delta=1 /(2 m(m-1))$ and observe that, for any $f \in \Phi$, if $x, y \in X \backslash A_{f}$ and $|h(x)-h(y)| \leqq \delta$, then we can find $i_{0} \in\{1, \ldots, m\}$ such that $x, y \in Z_{i_{0}} \cap P_{i_{0}}$, so $|f(x)-f(y)| \leqq \varepsilon$.

Now, we are in a position to prove the main theorem of this section.

1.7. Theorem. $T(\Phi)$ holds for any $\Phi \subset F[\mathscr{U}, \quad]$ if and only if $\left(\delta_{n}\right)$ is weakly (*)-regular.

Proof. To begin with, let us take $A \in \mathscr{M}$ and $n \in N$. Denote by $\psi_{A}$ the characteristic function of $A$ and observe that if $f \in C_{\mathrm{c}-\mathrm{o}}(X)$ is so chosen that $\{x \in X$ : $\left.\chi_{A}(x) \neq f(x)\right\} \in J_{n}$, then $A \Delta f^{-1}(1) \in j_{n}$.

Sufficiency. Let us assume that $\left(J_{n}\right)$ is weakly $(*)$-regular. Suppose that a family $\Phi \subset F[\mathscr{U}, j]$ is $\left(j_{n}\right)$-compact and take an arbitrary $n_{0} \in N$. Choose a sequence $\left(k_{i}\right)$ of positive integers such that $\bigcup_{i=1}^{\gamma_{j}} E_{i} \in \mho_{n_{0}}$ whenever $E_{i} \in \nu_{k_{i}}$ for $i \in N$. By Theorem 0.3 , there exists a positive integer $t$ such that $\{x \in X:|f(x)|>t\} \in J_{k_{1}}$ for any $f \in \Phi$. Applying Lemma 1.6, we find collections $\left\{A_{f}^{i}: f \in \Phi\right\} \subset j_{k_{i+1}}$, continuous functions $h_{i}: X \rightarrow[0,1]$ and real numbers $\delta_{i}>0$, such that, for any $i \in N$ and $f \in \Phi$, we have $|f(x)-f(y)| \leqq 1 / i$ whenever $x, y \in X \backslash A_{f}^{i}$ and $\left|h_{i}(x)-h_{i}(y)\right| \leqq \delta_{i}$. Let us put

$$
h(x, y)=\sum_{i=1}^{\infty} \frac{\left|h_{i}(x)-h_{i}(y)\right|}{2^{i}} \text { for } x, y \in X,
$$

and $A_{f}=\{x \in X:|f(x)|>t\} \cup \bigcup_{i=1}^{\infty} A_{f}^{i}$ for $f \in \Phi$. Obviously, $A_{f} \in \mathcal{J}_{n_{0}}$ for $f \in \Phi$. Following the construction carried out in the proof of Theorem 1 from [25] (cf. also [15; proof of Lemma 1, p. 228]), we can find a nondecreasing bounded uniformly continuous function $g:[0,+\infty) \rightarrow R$ such that $g(0)=0$ and $|f(x)-f(y)| \leqq$ $\leqq g(h(x, y))$ whenever $f \in \Phi$ and $x, y \in X \backslash A_{f}$. Let us define

$$
f^{*}(x)=\sup \left\{f(y)-g(h(x, y)): y \in X \backslash A_{f}\right\} \text { for } f \in \Phi \text { and } x \in X .
$$


Similarly as in [25; proof of Theorem 1] (cf. also [15; proof of Lemma 1]) we show that the family $\left\{f^{*}: f \in \Phi\right\}$ is equibounded and evenly continuous. To conclude the proof, it suffices to apply the Ascoli theorem (cf. [5; Th. 3.4.20]) and to observe that $\left\{x \in X: f(x) \neq f^{*}(x)\right\} \subset A_{f}$ for any $f \in \Phi$.

While the Baire sets can be defined in any topological space $X$, if $X$ is not completely regular, there is generally no meaningful relationship between $\mathscr{B}^{*}(X)$ and the topology of $X$; however, it is worth noticing that Theorem 1.7 remains true if $X$ is a Hausdorff $k$-space (not necessarily Tychonoff).

1.8. Corollary. If $\mathscr{U}=\mathscr{B}^{*}(X)$, then $\left(\sigma_{n}\right)$ is $(*)$-regular; hence $T(\Phi)$ holds for any $\Phi \subset F[\mathscr{M}, . j]$.

1.9. Corollary. If $X$ is normal and $\left(\sigma_{n}\right)$ is regular, then $\left(\sigma_{n}\right)$ is weakly $(*)$-regular; thus $T(\Phi)$ holds for any $\Phi \subset F[\mathscr{M}, 0]$.

Proof. Let us fix $A \in \mathscr{M}$ and $n \in N$. Take sets $F \in \mathscr{F}(X) \cap \mathscr{M}$ and $G \in \mathscr{G}(X) \cap \mathscr{M}$, such that $F \subset A \subset G$ and $G \backslash F \in J_{n}$. The normality of $X$ implies the existence of an $f \in C_{\mathrm{c}-\mathrm{o}}(X)$ such that $F \subset f^{-1}(0)$ and $X \backslash G \subset f^{-1}(1)$. Then $A \Delta f^{-1}(0) \subset$ $\subset G \backslash F$, hence $A \Delta f^{-1}(0) \in \mho_{n}$.

Let $\mathscr{H}$ be a family of subsets of $X$. We say that $A \subset X$ is Souslin- $\mathscr{H}$ if, for each $f \in N^{()}$and each $p \in N$, there are sets $H(f \uparrow p) \in \mathscr{H}$ depending only on $f \uparrow p$ (i.e. $H(f \nmid p)=H\left(g\lceil p)\right.$ whenever $f\left\lceil p=g\lceil p)\right.$ and such that $A=\bigcup_{f \in N^{\omega}} \bigcap_{p=1}^{\infty} H(f\lceil p)$ (cf. [22] and [7; p. 1002]).

The following lemma can be regarded as a special abstract version of Choquet's capacitability theorem (cf. [4; Th. 1, p. 84] and [7; Th. 10.8]).

1.10. Lemma. Suppose that $\mathscr{M}$ is stable under the Souslin operation. Let $\mathscr{H} \subset \mathscr{M}$ be a family which is closed under finite unions and countable intersections. Then, for each Souslin- $\mathscr{H}$ set $A \subset X$ and any $n \in N$, there exists an $H \subset A$ with $H \in \mathscr{H}$ and $A \backslash H \in \mathcal{J}_{n}$.

Proof. We shall use the same notation as in [7; proof of 10.8]. Let $A=$ $=\bigcup_{f \in N^{\omega}} \bigcap_{p=1}^{\infty} H\left(f\lceil p)\right.$ where $H\left(f\lceil p) \in \mathscr{H}\right.$ for any $f \in N^{(\omega)}$ and $p \in N$. We may assume that $(H(f \uparrow p))_{p \in N}$ is a decreasing sequence. If $p, n \in N$ and $s \in N^{p}$, let $\langle s, n\rangle \in N^{p+1}$ be such that $\langle s, n\rangle \uparrow p=s$ and $\langle s, n\rangle=n$; moreover, we define a map $\langle n\rangle: 1 \rightarrow N$ by letting $\langle n\rangle(0)=n$. For $f, g \in N^{\alpha}(\alpha \leqq \omega)$, we write $f \leqq g$ to denote that $f(n) \leqq$ $\leqq g(n)$ for each $n \in \alpha$. For $s \in N^{p}$, we set $A(s)=\bigcup\left\{\bigcap_{q=1}^{\infty} H\left(f\lceil q): f \in N^{\omega}, f\lceil p=s\}\right.\right.$ and $B(s)=\bigcup\left\{A(r): r \in N^{p}, \quad r \leqq s\right\}$. Then $A(s)=\bigcup_{\infty} A(\langle s, n\rangle)$ and $B(s)=$ $=\bigcup_{n=1}^{\infty} B(\langle s, n\rangle)$. Furthermore, $A=\bigcup_{n=1}^{\infty} A(\langle n\rangle)=\bigcup_{n=1}^{\infty} B(\langle n\rangle)$, and $B(r) \subset B(s)$ for each $r, s \in N^{p}$ with $r \leqq s$.

Take an $m \in N$ and choose $m_{0} \in N$ such that $E_{1} \cup E_{2} \in \sim_{m}$ whenever $E_{1}, E_{2} \in \sigma_{m}$. 
Inductively, we shall construct an $h \in N^{\omega}$ such that $A \backslash B\left(h\lceil p) \in \sigma_{m_{0}}\right.$ for each $p \in N$.

Let $\left(k_{i}\right)$ be a sequence of positive integers such that $\bigcup_{i=1}^{\infty} E_{i} \in \diamond_{m_{0}}$ whenever $E_{i} \in \diamond_{k_{i}}$ for $i \in N$. Since $A=\bigcup_{n=1}^{\infty} B(\langle n\rangle)$ and $B(\langle n\rangle) \subset B(\langle n+1\rangle)$, it follows from the u.s.c. of $\left(\mho_{n}\right)$ that there exists $n_{1} \in N$ with $A \backslash B\left(\left\langle n_{1}\right\rangle\right) \in \delta_{k_{1}}$. As $B\left(\left\langle n_{1}\right\rangle\right)=\bigcup_{n=1}^{\infty} B\left(\left\langle n_{1}, n\right\rangle\right)$ and $B\left(\left\langle n_{1}, n\right\rangle\right) \subset B\left(\left\langle n_{1}, n+1\right\rangle\right)$, the u.s.c. of $\left(\sigma_{n}\right)$ implies the existence of $n_{2} \in N$ with $B\left(\left\langle n_{1}\right\rangle\right) \backslash B\left(\left\langle n_{1}, n_{2}\right\rangle\right) \in \sigma_{k_{2}}$. In this way, we can inductively define a sequence $n_{1}, n_{2}, \ldots$ of positive integers such that $B\left(\left\langle n_{1}, \ldots, n_{p}\right\rangle\right) \backslash B\left(\left\langle n_{1}, \ldots, n_{p+1}\right\rangle\right) \in \Delta_{k_{p+1}}$ for any $p \in N$. Let $h(p-1)=n_{p}$ for $p \in N$. Then $B\left(h\lceil p)=B\left(\left\langle n_{1}, \ldots, n_{p}\right\rangle\right)\right.$ and $A \backslash B\left(h\lceil p) \subset\left[A \backslash B\left(\left\langle n_{1}\right\rangle\right)\right] \cup \bigcup_{i=1}\left[B\left(\left\langle n_{1}, \ldots, n_{i}\right\rangle\right) \backslash B\left(\left\langle n_{1}, \ldots, n_{i+1}\right\rangle\right)\right]\right.$, so $A \backslash B\left(h\lceil p) \in \circlearrowleft_{m_{0}}\right.$ for any $p \in N$. The set $H_{p}=\bigcup\left\{H(f \uparrow p): f \in N^{()}, f \leqq h\right\}(p \in N)$ belongs to $\mathscr{H}$; moreover, $A\left(f\lceil p) \subset A \cap H_{p}\right.$ for each $f \in N^{(\omega)}$ with $f \leqq h$ (cf. [7; proof of 10.8]). Consequently, $B\left(h\lceil p) \subset A \cap H_{p}\right.$, and hence, $A \backslash H_{p} \in \ddots_{m_{0}}$ for any $p \in N$. Put $H=\bigcap_{p=1}^{\infty} H_{p}$. Of course, $H \in \mathscr{H}$. Similarly as in [7; proof of 10.8$]$ we show that $H \subset A$. Since $H_{p+1} \subset H_{p}$ for any $p \in N$, it follows from the u.s.c. of $\left(s_{n}\right)$ that there exists $p_{0} \in N$ with $H_{p_{0}} \backslash H \in \sigma_{m_{0}}$. Then $A \backslash H \subset\left(A \backslash H_{p_{0}}\right) \cup\left(H_{p_{0}} \backslash H\right)$, so $A \backslash H \in J_{m}$.

1.11. Lemma. Let $\mathscr{A}$ denote the family of all sets $A \in \mathscr{M}$ having the property that, for any $n \in N$, there exists $F \in \mathscr{F}^{*}(X)$ with $A \Delta F \in ._{n}$. Then $\mathscr{A}$ forms a $\sigma$ algebra.

Proof. Let fix $n_{0} \in N$ and take a sequence $\left(k_{i}\right)$ of positive integers such that $\left.\bigcup^{\infty} E_{i} \in\right\lrcorner_{n_{0}}$ whenever $E_{i} \in J_{k_{i}}$ for $i \in N$. Consider any $A \in \mathscr{A}$. There exists $F \in \mathscr{F}^{*}(X)$ with $A \Delta F \in J_{k_{1}}$. By Lemma 1.4, we can find $H \in \mathscr{F}^{*}(X)$ such that $H \subset X \backslash F$ and $(X \backslash F) \backslash H \in s_{k_{2}}$. Then $(X \backslash A) \Delta H \subset(A \Delta F) \cup[(X \backslash F) \backslash H]$, so $(X \backslash A) \Delta H \in$ $\in \Im_{n_{0}}$ and $\mathscr{A}$ is stable under complements.

To show that $\mathscr{A}$ is stable under countable unions, let us take $A_{i} \in \mathscr{A}(i \in N)$ and choose $F_{i} \in \mathscr{F}^{*}(X)$ such that $A_{i} \Delta F_{i} \in \sigma_{x_{i+1}}$ for $i \in N$. As $\left(s_{n}\right)$ is u.s.c., there exists $m \in N$ with $\left(\bigcup_{i=1}^{\infty} F_{i}\right) \backslash\left(\bigcup_{i=1}^{m} F_{i}\right) \in \sigma_{k_{1}}$. Then $\left(\bigcup_{i=1}^{\infty} A_{i}\right) \Delta\left(\bigcup_{i=1}^{m} F_{i}\right) \subset \bigcup_{i=1}^{\infty}\left(A_{i} \Delta F_{i}\right) \cup$ $\cup\left[\left(\bigcup_{i=1}^{\infty} F_{i}\right) \backslash\left(\bigcup_{i=1}^{m} F_{i}\right)\right]$; therefore $\bigcup_{i=1}^{\infty} A_{i} \in \mathscr{A}$ because $\left(\bigcup_{i=1}^{\infty} A_{i}\right) \Delta\left(\bigcup_{i=1}^{m} F_{i}\right) \in \sigma_{n_{0}}$.

Let us recall that $\left.\mathscr{M}^{\wedge},(\lrcorner_{n}^{\wedge}\right)$ and $\jmath^{\wedge}$ denote the completions of $\mathscr{M},\left(\jmath_{n}\right)$ and $\lrcorner$, respectively (cf. Remark 0.5 ).

1.12. Theorem. Suppose that $\mathscr{M}=\mathscr{B}(X)$ has the following property:

(S) for any $A \subset X$, there exists $B \in \mathscr{M}^{\wedge}$ such that $A \subset B$ and $B \backslash C \in \sigma^{\wedge}$ whenever $C \in \mathscr{M}^{\wedge}$ and $A \subset C \subset B$.

If, for any $G \in \mathscr{G}(X)$ and $n \in N$, there exists a Souslin-F̈* $(X)$ set $H$ such that 
$G \Delta H \in \sigma_{n}$, then $\left(\sigma_{n}\right)$ is weakly $(*)$-regular; thus $T(\Phi)$ holds for any $\Phi \subset F[\mathscr{M}, 0]$. Proof. It follows from [22; Th. 2.9.2, p. 41] that $\mathscr{U}^{\wedge}$ is closed under the Souslin operation. For $G \in \mathscr{G}(X)$ and $n_{0} \in N$, take a Souslin- $\mathscr{F} *(X)$ set $H$ such that $G \Delta H \in$ $\in \varsigma_{m}$ where $m \in N$ is so chosen that $A \cup B \in \varsigma_{n_{0}}$ whenever $A, B \in \varsigma_{m}$. By Lemma 1.10, there exists $F \in \mathscr{F}^{*}(X)$ such that $F \subset H$ and $H \backslash F \in \jmath_{m}$. Then $G \Delta F \subset(G \Delta H) \cup$ $\cup(H \backslash F)$, so $G \Delta F \in \sigma_{n_{0}}$. Using Lemma 1.11 , we obtain that $\left(\diamond_{n}\right)$ is weakly $(*)$ regular.

1.13. Theorem. Suppose that $\mathscr{M}=\mathscr{B}(X)$ has the property $(\mathrm{S})$ defined in Theorem

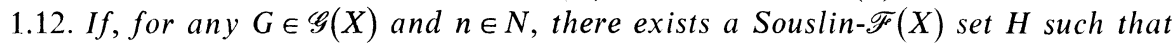
$H \subset G$ and $G \backslash H \in\lrcorner_{n}$, then $\left(\varsigma_{n}\right)$ is regular. Consequently, if we additionally assume that $X$ is normal, then $T(\Phi)$ holds for any $\Phi \subset F[\mathscr{M}, 0]$.

Proof. Arguing similarly as in the proof of Theorem 1.12, we deduce that, for any $G \in \mathscr{G}(X)$ and $n \in N$, there exists $F \in \mathscr{F}(X)$ with $F \subset G$ and $G \backslash F \in s_{n}$. Following the proof of Lemma 1.4, we show that (\lrcorner$\left._{n}\right)$ is regular. The proof will be completed if we apply Corollary 1.9 .

To obtain one more consequence of Theorem 1.7, we need the following

1.14. Definition. (cf. [7; Def. 2.3]). The small system $\left(\diamond_{n}\right)$ is called $\tau$-additive (weakly $\tau$-additive, respectively) if any family $\mathscr{V} \subset \mathscr{G}^{*}(X)$, such that $\bigcup\{V: V \in \mathscr{V}\} \in$ $\in \mathscr{M}$ (respectively $\bigcup\{V: V \in \mathscr{V}\}=X$ ), has the property that, for each $n \in N$, there exists a finite subfamily $\mathscr{U}\}$ of $\mathscr{V}$ such that the set $(\bigcup\{V: V \in \mathscr{V}\}) \backslash U\{V: V \in \mathscr{U}\}$ is a member of $\sigma_{n}$.

1.15. Proposition. If $\mathscr{M}=\mathscr{B}^{*}(X)$, then the weak $\tau$-additivity of $\left(j_{n}\right)$ is equivalent to the $\tau$-additivity of $\left(\diamond_{n}\right)$.

Proof. Suppose that $\left(\varsigma_{n}\right)$ is weakly $\tau$-additive. Let $E \in \mathscr{B}^{*}(X)$ and let $E=$ $=\bigcup\{V: V \in \mathscr{V}\}$ for some $\mathscr{V} \subset \mathscr{G}^{*}(X)$. Fix $n_{0} \in N$ and take $m \in N$ such that $A \cup B \in$ $\in \sigma_{n_{0}}$ whenever $A, B \in \varsigma_{m}$. By Lemma 1.4, there is $F \in \mathscr{F}^{*}(X)$ with $F \subset E$ and $E \backslash F \in$ $\in J_{m}$. Then $X=\bigcup\{V \cup(X \backslash F): V \in \mathscr{V}\}$, so there exists a finite family $\mathscr{U} \subset \mathscr{V}$. with the set $H=X \backslash \bigcup\{V \cup(X \backslash F): V \in \mathscr{U}\}$ belonging to $\varsigma_{m}$. Then $E \backslash \bigcup\{V$ : $V \in \mathscr{U}\} \subset H \cup(E \backslash F)$, hence $E \backslash \bigcup\{V: V \in \mathscr{U}\}$ is a member of $\jmath_{n_{0}}$.

1.16. Theorem. Suppose that $\mathscr{M}=\mathscr{B}(X)$. If $\left(\sigma_{n}\right)$ is $\tau$-additive, then it is weakly (*)-regular; consequently, $T(\Phi)$ holds for any $\Phi \subset F[\mathscr{M}, 0]$.

Proof. Consider any $G \in \mathscr{G}(X)$ and $n_{0} \in N$. Choose $m \in N$ such that $A \cup B \in \supset_{n_{0}}$ whenever $A, B \in \jmath_{m}$. As the space $X$ is Tychonoff, there exists a family $\mathscr{V} \subset \mathscr{G}^{*}(X)$ such that $G=\bigcup\{V: V \in \mathscr{V}\}$. By the $\tau$-additivity of $\left(\varsigma_{n}\right)$, we can find a finite subfamily $\mathscr{U}$ of $\mathscr{V}$ such that $G \backslash H \in \varsigma_{m}$ where $H=\bigcup\{V: V \in \mathscr{U}\}$. By Lemma 1.4, there exists $F \in \mathscr{F} *(X)$ with $F \subset H$ and $H \backslash F \in \sigma_{m}$. Then, since $G \backslash F \subset(G \backslash H) \cup(H \backslash F)$, we have $G \backslash F \in \sigma_{n_{0}}$. Lemma 1.11 implies the weak $(*)$-regularity of $\left(\delta_{n}\right)$.

Let us conclude studying the implication $T(\Phi)$ with the observation that various abstract versions of the Lusin theorem on approximation of a measurable function to a continuous function can easily be deduced from the results of this section. In 
particular, we obtain the following generalization of Theorems 3 and 4 of [21] (cf. also [17; Th. 18.36]):

1.17. Theorem. Let $\left(\delta_{n}\right)$ be a weakly $(*)$-regular u.s.c. small system on a $\sigma$-algebra $\mathscr{M}$ of subsets of a topological space $X$ (not necessarily a Tychonoff $k$-space) such that $\mathscr{B}^{*}(X) \subset \mathscr{M}$. Then an $\lrcorner^{\wedge}$-a.e. finite function $f: X \rightarrow R \cup\{ \pm \infty\}$ is $\mathscr{M}^{\wedge}$ measurable if and only if, for any $n \in N$, there exists a continuous function $f_{n}: X \rightarrow R$ with $\left\{x \in X: f(x) \neq f_{n}(x)\right\} \in \mathcal{J}_{n}$.

The proof of the above theorem is similar to that of Theorem 1.7. Obviously, the assumption of the weak $(*)$-regularity of $\left(\sigma_{n}\right)$ is necessary in Theorem 1.17.

\section{THE IMPLICATION $T^{-1}(\Phi)$}

2.1. Theorem. If $\left(\jmath_{n}\right)$ is weakly $\tau$-additive, then $T^{-1}(\Phi)$ holds for any $\Phi \subset$ $\subset F[\mathscr{M}, j]$.

Proof. Let $\Phi \subset F[\mathscr{M}, J]$ have the property that, for any $m \in N$, there exists a compact set $\Phi_{m} \subset C_{\mathrm{c}-\mathrm{o}}(X)$ such that to each $f \in \Phi$ we can assign some $f_{m} \in \Phi_{m}$ with $\left\{x \in X: f(x) \neq f_{m}(x)\right\} \in J_{m}$. We shall show that $\Phi$ is $\left(\sigma_{n}\right)$-equibounded and $\left(\sigma_{n}\right)$ equimeasurable (cf. Theorem 0.3 ).

Let us fix $n_{0} \in N$ and $\varepsilon>0$. Choose $m \in N$ such that $A \cup B \in \sigma_{n_{0}}$ whenever $A, B \in$ $\in \delta_{m}$. As $\Phi_{m}$ is compact in $C_{\mathrm{c}-\mathrm{o}}(X)$, it follows from the Ascoli theorem (cf. [5; Th. 3.4.20]) that, for each $x \in X$, there exist a set $V_{x} \in \mathscr{G}^{*}(X)$ and a positive integer $t_{x}$, such that $x \in V_{x}$ and, for any $f \in \Phi$ and $y \in V_{x}$, we have $\left|f_{m}(y)\right| \leqq t_{x}$ and $\left|f_{m}(x)-f_{m}(y)\right| \leqq \varepsilon / 2$. The weak $\tau$-additivity of $\left(\sigma_{n}\right)$ implies that there exists a finite subcollection $\left\{V_{x_{1}}, \ldots, V_{x_{k}}\right\}$ of $\left\{V_{x}: x \in X\right\}$ such that the set $A=X \backslash \bigcup_{i=1}^{k} V_{x_{i}}$ belongs to $\sigma_{m}$. Let us put $t=\max \left\{t_{x_{1}}, \ldots, t_{x_{k}}\right\}$ and $A_{f}=A \cup\left\{x \in X: f(x) \neq f_{m}(x)\right\}$ for $f \in \Phi$. Of course, for any $f \in \Phi$, we have $A_{f} \in \delta_{n_{0}},\{x \in X:|f(x)|>t\} \subset A_{f}$ and $|f(x)-f(y)| \leqq \varepsilon$ whenever $x, y \in V_{x_{i}} \backslash A_{f}(i=1, \ldots, k)$. Therefore $\Phi$ is $\left(\iota_{n}\right)$ equibounded and $\left(\varsigma_{n}\right)$-equimeasurable. In view of Theorem $0.3, \Phi$ is $\left(\diamond_{n}\right)$-compact.

2.2. Proposition. If $X$ has the Lindelof property, then $\left(\sigma_{n}\right)$ is weakly $\tau$-additive; consequently, $T^{-1}(\Phi)$ holds for any $\Phi \subset F[\mathscr{M}, \jmath]$.

Proof. It suffices to observe that if $\mathscr{U} \subset \mathscr{G}^{*}(X)$ is a countable cover of $X$, then, since $\left(\sigma_{n}\right)$ is u.s.c., for any $n \in N$, we can choose a finite collection $\mathscr{W} \subset \mathscr{U}$ such that the set $X \backslash \cup\{W: W \in \mathscr{W}\}$ belongs to $s_{n}$.

2.3. Lemma. Let $n_{0} \in N$ be fixed. Any point-finite family $\mathscr{A} \subset \mathscr{M} \backslash \jmath_{n_{0}}$ is finite. Proof. Suppose $\mathscr{A} \subset \mathscr{M} \backslash \sigma_{n_{0}}$ is infinite. Let $\left\{A_{1}, A_{2}, \ldots\right\}$ be an infinite countable subfamily of $\mathscr{A}$. Since $\bigcup_{i=n}^{\infty} A_{i} \notin \sigma_{n_{0}}$ for any $n \in N$, the u.s.c. of $\left(\mho_{n}\right)$ implies that $\emptyset \neq$ $\neq \bigcap_{n=1}^{\infty} \bigcup_{i=n}^{\infty} A_{i}$, so $\mathscr{A}$ cannot be point-finite. 
2.4. Proposition. Any point-finite family $\mathscr{A} \subset \mathscr{M} \backslash j$ is countable. In particular, any family of pairwise disjoint members of $\mathscr{M} \backslash \mathrm{s}$ is countable, i.e. the pair $(\mathscr{M}, \sigma)$ satisfies the countable chain condition.

2.5. Proposition. If $X$ is paracompact and the set $\bigcup\left\{G \in \mathscr{G}^{*}(X): G \in \delta\right\}$ belongs to $\dot{\nu}^{\wedge}$, then $\left(j_{n}\right)$ is weakly $\tau$-additive, so $T^{-1}(\Phi)$ holds for any $\Phi \subset F[\mathscr{M}, \jmath]$.

Proof. Consider any cover $\mathscr{V} \subset \mathscr{G}^{*}(X)$ of $X$ and any $n_{0} \in N$. Let $\mathscr{U} \subset \mathscr{G}^{*}(X)$ be a locally finite refinement of $\mathscr{V}$. By virtue of Proposition 2.4, the family $\mathscr{U} \backslash \mathrm{s}$ is countable. Since $\left(j_{n}\right)$ is u.s.c., there exists a finite family $\mathscr{W} \subset \mathscr{U} \backslash s$ such that the set $A=\left(\bigcup\left\{U: U \in \mathbb{M}^{\prime}\right\}\right) \backslash \bigcup\{W: W \in \mathscr{W}\}$ belongs to $n_{n_{0}}$. To each $W \in \mathscr{W}$ assign some $V_{W} \in \mathscr{Y}^{-}$with $W \subset V_{W}$, and put $B=\bigcup\left\{V_{W}: W \in \mathscr{W}\right\}$. Then $X \backslash B \subset A \cup$ $\cup \bigcup\{U \in \mathbb{U}: U \in$.$\} , so X \backslash B \in{ }_{n_{n_{0}}}$. This implies that $X \backslash B \in \triangleleft_{n_{0}}$ because $X \backslash B \in \mathscr{M}$. Hence $\left(s_{n}\right)$ is weakly $\tau$-additive.

Since each open cover of a metacompact space has a point-finite open refinement, applying Proposition 2.4 and arguing similarly as in the proof of Proposition 2.5, we obtain the following

2.6. Proposition. Suppose that $\mathscr{M} \subset \mathscr{B}(X)$ and $X$ is metacompact. If the set $\bigcup\{G \in \mathscr{G}(X): G \in J\}$ belongs to $\delta$, then $\left(\delta_{n}\right)$ is weakly $\tau$-additive; thus $T^{-1}(\Phi)$ holds for any $\Phi \subset F[., 1$,$] .$

It is known that, under the assumption of $\mathrm{MA}+7 \mathrm{CH}$, every locally compact metalindelöf space satisfying the countable chain condition is Lindelöf (cf. [7; Coroll. 4.9]). Using this property, we shall prove

2.7. Proposition (MA $+\neg \mathrm{CH}$ ). Let $X$ be a locally compact metalindelöf space. If $\mathscr{M} \supset \mathscr{B}(X)$ and the set $\bigcup\{G \in \mathscr{G}(X): G \in \delta\}$ belongs to $s$, then $\left(\delta_{n}\right)$ is weakly $\tau$-additive; consequently, $T^{-1}(\Phi)$ holds for any $\Phi \subset F[\mathscr{M}, \jmath]$.

Proof. Denote $A=\bigcup\{G \in \mathscr{G}(X): G \in J\}$ and $Y=X \backslash A$. Of course, $Y$ is a locally compact metalindelöf subspace of $X$. By Proposition 2.4, $Y$ satisfies the countable chain condition, hence $Y$ is Lindelöf. If $\mathscr{V}$ is an open cover of $X$, then there exists a countable subfamily $\mathscr{U}$ of $\mathscr{V}$ which covers $Y$. For any $n \in N$, we can find a finite family $\mathscr{W} \subset \mathbb{U}$ such that the set $(\bigcup\{U: U \in \mathscr{U}\}) \backslash \bigcup\{W: W \in \mathscr{W}\}$ belongs to $o_{n}$; then $X \backslash \bigcup\{W: W \in \mathscr{W}\}$ is a member of $\sigma_{n}$.

We shall say that a cardinal $\varkappa$ carries a $\sigma$-saturated $\sigma$-ideal if there exists a $\sigma$-ideal $\mathscr{L}$ on the $\sigma$-algebra $\mathscr{P}(\varkappa)$ of all subsets of $\chi$, such that each countable subset of $\varkappa$ belongs to $\mathscr{L}, \varkappa \notin \mathscr{L}$ and any family of pairwise disjoint members of $\mathscr{P}(x) \backslash \mathscr{L}$ is countable.

A cardinal $\varkappa$ will be called upper semicontinuous if there exists an upper semicontinuous small system $\left(\mathscr{L}_{n}\right)$ on $\mathscr{P}(\varkappa)$ such that $\chi \notin \bigcap_{n=1}^{\infty} \mathscr{L}_{n}$ and $\bigcap_{n=1}^{\infty} \mathscr{L}_{n}$ contains each
countable subset of $\chi$.

It follows from Proposition 2.4 that every upper semicontinuous cardinal carries a $\sigma$-saturated $\sigma$-ideal. Of course, every real-valued measurable cardinal (cf. [7; p. 972] and [12]) is upper semicontinuous. Let us mention that in the ZFC set 
theory the statements "there exists a real-valued measurable cardinal" and "there exists a cardinal which carries a $\sigma$-saturated $\sigma$-ideal" are equiconsistent (i.e. if one is consistent with ZFC, then so is the other) (cf. [12]). K. L. Prikry proved in [19] that if the existence of a real-valued measurable cardinal is consistent with ZFC, then it is consistent with ZFC that there exists a cardinal which carries a $\sigma$-saturated $\sigma$-ideal without being real-valued measurable.

Let us recall that $X$ is a weakly $\theta$-refinable space if each open cover of $X$ has an open refinement $\bigcup_{n=1}^{\infty} \mathscr{U}_{n}$ such that, for any $x \in X$, there is a positive integer $n_{x}$ with $1 \leqq\left|\left\{U \in \mathscr{U}_{n_{x}}: x \in U\right\}\right|<\omega(\mathrm{cf} .[1])$.

2.8. Theorem (cf. [7; Th. 10.2]). Let $X$ be a weakly $\theta$-refinable space. If $\mathscr{M} \supset$ $\supset \mathscr{B}(X)$, and $X$ contains no discrete subspace of upper semicontinuous cardinality (in particular, if $X$ contains no discrete subspace of cardinality carrying a $\sigma$ saturated $\sigma$-ideal), then $\left(\sigma_{n}\right)$ is weakly $\tau$-additive; consequently, $T^{-1}(\Phi)$ holds for any $\Phi \subset F[\mathscr{M}, 0]$.

Proof. Let us consider any open cover $\mathscr{V}$ of $X$ and any $n_{0} \in N$. By [1; Th. 3.6], $\infty$ we can find families $\mathscr{V}_{i} \subset \mathscr{G}(X)$ such that $\bigcup_{i=1}^{\infty} \mathscr{V}_{i}$ is a refinement of $\mathscr{V}$ and, for each $x \in X$, there is a positive integer $i_{x}$ with $\left|\left\{V \in \mathscr{V}_{i_{x}}: x \in V\right\}\right|=1$. If $X_{i}=\{x \in X$ : $\left.\left|\left\{V \in \mathscr{V}_{i}: x \in V\right\}\right|=1\right\}$ for $i \in N$, then $X=\bigcup_{i=1}^{\infty} X_{i}$. Furthermore, since the sets $\{x \in X$ : $\left.\left|\left\{V \in \mathscr{V}_{i}: x \in V\right\}\right| \geqq k\right\}(i, k \in N)$ are open in $X$, we have $X_{i} \in \mathscr{B}(X)$ for $i \in N$. Let us put $Y_{i}=\bigcup\left\{V \cap X_{i}: V \in \mathscr{V}_{i}\right.$ and $\left.V \cap X_{i} \in \delta\right\}$ for $i \in N$ and suppose that $Y_{i} \in$, for any $i \in N$. In view of Proposition 2.4, each of the families $\mathscr{W}_{i}=\left\{V \cap X_{i}: V \in \mathscr{V}_{i}\right.$ and $\left.V \cap X_{i} \notin \delta\right\}$ is countable, so, by the u.s.c. of $\left(\delta_{n}\right)$, there exists a finite family $\mathscr{W} \subset \bigcup_{i=1}^{\infty} \mathscr{W}_{i}$ such that the set $E=\left(\bigcup\left\{W: W \in \bigcup_{i=1}^{\infty} \mathscr{W}_{i}\right\}\right) \backslash \bigcup\{W: W \in \mathscr{W}\}$ belongs to $\sigma_{n_{0}}$. To each $W \in \mathscr{W}$ assign some $V_{W} \in \mathscr{V}$ with $W \subset V_{W}$. Then the set $F=$ $=X \backslash \bigcup\left\{V_{W}: W \in \mathscr{W}\right\}$ is a member of $\delta_{n_{0}}$ because $F \subset E \cup \bigcup_{i=1}^{\infty} Y_{i}$.

Suppose now that $Y_{i_{0}} \notin$ ofor some $i_{0} \in N$. Since the family $\mathscr{P}=\left\{V \cap X i_{i_{0}}: V \in \mathscr{V}_{i_{0}}\right.$ and $\left.\emptyset \neq V \cap X_{i_{0}} \in \sigma\right\}$ consists of nonempty pairwise disjoint open subsets of $X_{i_{0}}$, by assigning to each $P \in \mathscr{P}$ some $x_{P} \in P$, we obtain a discrete subspace $D=$ $=\left\{x_{P}: P \in \mathscr{P}\right\}$ of $X$. The proof of the weak $\tau$-additivity of $\left(\varsigma_{n}\right)$ will be completed by showing that the cardinality of $D$ is upper semicontinuous. Bearing this in mind, let us define

$$
\circlearrowleft_{n}^{*}=\left\{A \subset D: \bigcup\left\{P \in \mathscr{P}: x_{P} \in A\right\} \in o_{n}\right\} \text { for } n \in N \text {. }
$$

Without any difficulties one checks that $\left(\sigma_{n}^{*}\right)$ forms a small system on $\mathscr{B}(D)$. If $\left(A_{n}\right)$ is a nonincreasing sequence of members of $\mathscr{B}(D) \backslash o_{m_{0}}^{*}$ for some $m_{0} \in N$, then letting $B_{n}=\bigcup\left\{P \in \mathscr{P}: x_{P} \in A_{n}\right\}$ for $n \in N$, we obtain a nonincreasing sequence $\left(B_{n}\right)$ of 
members of $\mathscr{B}(X) \backslash \sigma_{m_{0}}$. As $\emptyset \neq \bigcap^{\infty} B_{n}$ and any two distinct sets from $\mathscr{P}$ are disjoint, we have $\emptyset \neq \bigcap_{n=1}^{\infty} A_{n}$; hence $\left(\delta_{n}^{*}\right)^{n=1}$ is u.s.c. Obviously, $\bigcap_{n=1}^{\infty} \delta_{n}^{*}$ contains each countable subset of $D$ for $\mathscr{P} \subset \jmath$; moreover $D \notin \bigcap_{n=1} J_{n}^{*}$ because $Y_{i} \notin \jmath$. Consequently, $|D|$ is upper semicontinuous.

Finally, let us note that only those parts of our theorems to whose proofs the Ascoli theorem is applied require $X$ to be a $k$-space.

\section{OPEN PROBLEMS}

3.1. Question. Do there exist a Tychonoff $k$-space $X$ and an u.s.c. small system $\left(\nu_{n}\right)$ on $\mathscr{B}(X)$, such that $T^{-1}(\Phi)$ holds for any $\Phi \subset F\left[\mathscr{B}(X), \bigcap_{n=1}^{\infty} \nu_{n}\right]$ but $\left(\vartheta_{n}\right)$ is not
weakly $\tau$-additive?

3.2. Question. Can the assumption of the regularity of $\left(\sigma_{n}\right)$ be replaced by the assumption of the weak regularity of $\left(J_{n}\right)$ in Corollary 1.9?

3.3. Question. Does Theorem 2.8 remain true if we replace the assumption that $X$ contains no discrete subspace of upper semicontinuous cardinality by the assumption that none of discrete subspaces of $X$ is of real-valued measurable cardinality?

3.4. Question. Is every upper semicontinuous cardinal real-valued measurable?

3.5. Question. If a cardinal $\varkappa$ carries a $\sigma$-saturated $\sigma$-ideal, is $\varkappa$ upper semicontinuous?

3.6. Question. Does there exist a weakly Borel measure-complete Tychonoff space $X$ (cf. [7; Def. 7.1]) such that some u.s.c. small system on $\mathscr{B}(X)$ is not weakly $\tau$-additive?

3.7. Question. Does there exist a measure-compact space $X$ (cf. [7; Def. 14.3]) such that some u.s.c. small system on $\mathscr{B}^{*}(X)$ is not $\tau$-additive?

3.8. Question. What are necessary and sufficient conditions for a $\sigma$-ideal $s$ on a $\sigma$-algebra $\mathscr{M}$ of subsets of a set $X$ in order that an u.s.c. small system $\left(\sigma_{n}\right)$ on $\mathscr{M}$ exist, such that $\left.J=\bigcap_{n=1}^{\infty}\right\lrcorner_{n}$ ?

3.9. Question. What are necessary and sufficient conditions for a $\sigma$-ideal $j$ on the Borel $\sigma$-algebra $\mathscr{B}(X)$ of a Tychonoff space $X$ in order that a regular $((*)$-regular, weakly regular, weakly $(*)$-regular, $\tau$-additive and weakly $\tau$-additive, resp.) u.s.c. small system $\left(o_{n}\right)$ on $\mathscr{B}(X)$ exist, such that $\jmath=\bigcap_{n=1}^{\infty} o_{n}$ ?

3.10. Question. Do there exist a $\sigma$-algebra $\mathscr{M}$ of subsets of a set $X$ and an u.s.c. 
small system $\left(s_{n}\right)$ on $\mathscr{M}$, such that, for any finite $\sigma$-additive measure $\mu$ on $\mathscr{M}$, we have $\{A \in \mathscr{U}: \mu(A)=0\} \neq \bigcap_{n=1}^{\infty} \diamond_{n}$ ?

3.11. Question. What are necessary and sufficient conditions for an u.s.c. small system $\left(._{n}\right)$ on a $\sigma$-algebra $\mathscr{M}$ of subsets of a set $X$ in order that a finite $\sigma$-additive measure $\mu$ on $\mathscr{M}$ exist, such that $\{A \in \mathscr{M}: \mu(A)=0\}=\bigcap_{n=1}^{\infty} \circlearrowleft_{n}$ ?

It is not difficult to see that if $\left(._{n}\right)$ is an u.s.c. small system on a $\sigma$-algebra $\mathscr{M}$ and if $j^{*} \subset \mathscr{U}$ is a $\sigma$-ideal on $\mathscr{M}$ that contains $s=\bigcap_{n=1}^{\infty} j_{n}$, then, by putting

$$
\varkappa_{n}^{*}=\left\{A \cup B: A \in \sigma_{n} \text { and } B \in s^{*}\right\} \text { for } n \in N \text {, }
$$

we obtain an u.s.c. small system $\left(J_{n}^{*}\right)$ on $\mathscr{M}$ such that $\sigma^{*}=\bigcap_{n=1}^{\infty} J_{n}^{*}$. In connection with the above remark one can ask the following

3.12. Question. Suppose that $\delta$ and $\delta^{*}$ are $\sigma$-ideals on a $\sigma$-algebra $\mathscr{M}$ of subsets of a set $X$. If $j \subset j^{*}$ and $j=\{A \in \mathscr{M}: \mu(A)=0\}$ for some finite $\sigma$-additive measure $\mu$ on $\mathscr{M}$, does there exist a finite $\sigma$-additive measure $\mu^{*}$ on $\mathscr{M}$ for which $\diamond^{*}=\{A \in \mathscr{U}$ : $\mu^{*}(A)=0$ ?

Most of our questions are related to the general problem of the existence of a finite strictly positive measure on a Boolean algebra (cf. e.g. $[11,14,16,20,23,24])$.

\section{References}

[1] D. K. Burke: Covering properties, in: Handbook of Set-Theoretic Topology (K. Kunen, J. E. Vaughan, eds.), North Holland-Amsterdam, 1984, 347-422.

[2] F. Cafiero: Misura e Integrazione, Rome, 1959, 308-315.

[3] P. Capek: On small systems, Acta Fac. Rerum Natur. Univ. Comenian Math. 34 (1979), 93-101.

[4] G. Choquet: Forme abstraite du théorème de capacitabilité, Ann. Inst. Fourier 9 (1959), $83-89$.

[5] R. Engelking: General Topology, Warsaw, 1977.

[6] M. Fréchet: Sur les ensembles compacts de fonctions measurables, Fund. Math. 9 (1927), $25-32$.

[7] R.J. Gardner and W. F. Pfeffer: Borel measures, in: Handbook of Set-Theoretic Topology (K. Kunen, J. E. Vaughan, eds.), North Holland-Amsterdam, 1984, 961-1043.

[8] L. Gillman and M. Jerison: Rings of Continuous Functions, New York-Heidelberg-Berlin, 1976.

[9] E. H. Hanson: A note on compactness, Bull. Amer. Math. Soc. 39 (1933), 397-400.

[10] J. Hejduk and E. Wajch: Compactness in the sense of the convergence with respect to a small system, Math. Slov. 39 (1989), 267-275.

[11] A. Horn and A. Tarski: Measures in Boolean algebras, Trans. Amer. Math. Soc. 64 (1948), $467-497$. 
[12] T. Jech: Set Theory, New York, 1978.

[13] R. A. Johnson, J. Niewiarowski and T. Świqtkowski: Small systems convergence and metrizability, Proc. Amer. Math. Soc. 103 (1988), 105-112.

[14] J. L. Kelley: Measures on Boolean algebras, Pacific J. Math. 9 (1959), 1165-1177.

[15] J. Kisyński: Sur les familles compactes de fonctions mesurables, Colloq. Math. 7 (1960), $221-235$.

[16] D. Maharam: An a!gebraic characterization of measure algebras, Annals of Math. 48 (1947), 154-167.

[17] T. Neubrunn and B. Riečan: Miera a Integrál, Bratislava, 1981, 485-497.

[18] J. Niewiarowski: Convergence of sequences of real functions with respect to small systems, Math. Slov. 38 (1988), 333-340.

[19] K. Prikry: Changing measures into accessible cardinals, Dissert. Math. 68 (1970), 1-52.

[20] K. Prikry: On measures on complete Boolean algebras, J. Symb. Logic 36 (1971), 395-406.

[21] B. Riečan: Abstract formulation of some theorems of measure theory, Mat. Fyz. Časopis SAV 16 (1966), 268-273.

[22] C. A. Rogers and J. E. Jayne: $K$-analytic sets, in: Analytic Sets (C. A. Rogers, J. E. Jayne, eds.), London, 1980, 1-181.

[23] D. A. Vladimirov: On the existence of invariant measures on Boolean algebras, Dokl. Akad. Nauk SSSR 157 (1964), 764-766.

[24] D. A. Vladimirov: Invariant measures on Boolean algebras, Mat. Sb. (N.S.) 67 (109) (1965), $440-460$.

[25] E. Wajch: On small systems and compact families of Borel functions, Math. Slov. 40 (1990), $63-69$.

Author's address: Institute of Mathematics, University of Łódż, S. Banacha 22, 90-238 Łódż, Poland. 